\title{
Combined Analysis of Anti SARS-CoV-2 IgG and IgM Responses in COVID19 Patients in India
}

\author{
Mohana Kumari Chidananda ${ }^{1}$ - Deepy Zohmangaihi ${ }^{1} \cdot$ Sant Ram $^{1} \cdot$ \\ Shiv Lal Soni ${ }^{2} \cdot$ Vikas Suri $^{3} \cdot$ Pankaj Malhotra $^{3} \cdot$ Ravjit Singh Jassal $^{1}$. \\ Jyotdeep Kaur ${ }^{1} \cdot$ Indu Verma $^{1}$ (D) Sadhna Sharma ${ }^{1}$
}

Received: 9 March 2021 / Accepted: 3 June 2021 / Published online: 18 June 2021

(C) Association of Clinical Biochemists of India 2021, corrected publication 2021

\begin{abstract}
Coronavirus disease 2019 (COVID-19) caused by Severe Acute Respiratory Syndrome Coronavirus 2 (SARS-CoV-2) is a global health problem, India being the second most affected country. The kinetics of antibody response to SARS-CoV-2 in Indian population is not studied yet. To understand serological response in relation to age, gender, time period and severity of disease, Roche Elecsys anti-SARS-CoV-2 test was used which analysed both IgM and IgG. One hundred and three COVID-19 patients were enrolled. Seropositivity was seen in $64 \%$ of patients, with $33 \%$ at $\leq 7$ days, $62 \%$ between 8 and 15 days and $81 \%$ at $\geq 16$ days from the time of admission. Men $(65 \%)$ showed higher antibody response than women $(59 \%)$, whereas no difference was observed in seropositivity with respect to age of the patients. Dynamics of antibody responses revealed individual variations. Patients in ICU had higher antibody reactivity with $67 \%$ positivity as compared to $60 \%$ positivity in non-ICU patients. Kinetics of antibody response during COVID-19 disease varied in relation to gender, age, time period and severity and these factors might play an important role in treatment and control of COVID-19.
\end{abstract}

Jyotdeep Kaur

jyotdeep2001@yahoo.co.in

Indu Verma

induvermabio@gmail.com

1 Department of Biochemistry, Post Graduate Institute of Medical Education and Research, Chandigarh, India

2 Department of Anesthesia and Intensive Care, Post Graduate Institute of Medical Education and Research, Chandigarh, India

3 Department of Internal Medicine, Post Graduate Institute of Medical Education and Research, Chandigarh, India
Keywords COVID-19 · Anti SARS CoV-2 - Antibody response $\cdot$ SARS CoV-2

\section{Introduction}

Coronavirus disease 2019 (COVID-19), a pandemic of the year 2020, caused by Severe Acute Respiratory Syndrome Coronavirus 2 (SARS-CoV-2) of the family Coronaviridae, genus betacoronaviruses, has affected 216 countries in the world as declared by World Health Organisation (WHO) [1]. SARS-CoV-2 has 4 structural proteins-spike (S), envelope (E), membrane (M), and nucleocapsid (N) [2]. To date, six coronaviruses have been known to affect the human race namely MERS-CoV, SARS-CoV, $\mathrm{HCoV}$ NL63, HCoV-229E, HCoV-HKU1, and HCoV-OC43 [3]. According to WHO, $20 \%$ of the people affected with COVID-19 require hospitalization, and the rest of them recover with mild symptoms (https://www.who.int/emer gencies/diseases/novel-coronavirus-2019). The most common symptoms are fever, tiredness, dry cough, and less common symptoms being headache, nasal congestion, diarrhea, loss of taste and smell, sore throat, conjunctivitis, etc. [4]. Elderly, individuals with a previous medical history of diabetes mellitus, hypertension, cancer, respiratory disorders, and other chronic diseases are more prone to develop severe acute respiratory distress syndrome leading to intensive care treatment and mortality [5]. The mortality rate of COVID-19 is 2.8\% as reported by WHO (https:// www.who.int/emergencies/diseases/novel-coronavirus-

2019). Once exposed to the virus, the host immune system is activated and antiviral antibodies are produced. However, the antibody response to SARS-Co-V2 is poorly understood. Analyzing kinetics of antibody response will not only be important for diagnosis but also crucial to 
determining the extent of spread in the population for epidemiological studies, determining the previous exposure to the virus, and identification of convalescent plasma donors for passive immune therapy [6]. Serological responses vary in different age groups and gender, as well as disease severity and much information is not available on the effect of these variables on the antibody kinetics in COVID-19 patients. We, therefore, analyzed the kinetics of serological response in different age groups, men versus women and ICU versus non-ICU patients. The reported findings suggested that IgM is produced as early as 7 days and IgG by $10-15$ days after the onset of the symptoms with maximum seroconversion for IgM occurring at 4 weeks and for IgG at 6 weeks [3]. As the increase in the concentration of $\operatorname{IgM}, \operatorname{IgG}$, and sequence of rising are inconsistent in COVID patients [7-9] it would be advantageous to have a serological technique that includes detection of both IgM and IgG. In the present study, Roche Elecsys anti-SARS-CoV-2, a serological test used for qualitative identification of $\operatorname{IgM}$ and $\operatorname{IgG}$ antibodies against SARS-CoV-2 in humans, consisting of recombinant protein representing the structural antigen nucleocapsid $(\mathrm{N})$ has been used.

\section{Methodology}

\section{Study Design}

Retrospective study.

\section{Study Population}

COVID-19 positive patients confirmed by RT-PCR were recruited from Nehru Extension Block, Postgraduate Institute of Medical Education and Research (PGIMER), Chandigarh. The population taken for the study included males or females aged between 6 and 80 years. The study plan was approved by Institute Ethics Committee (Ref NoNK/6384/study/231). A total of 103 patients of COVID-19 were recruited for the study. Day of admission was considered as day 1 and variations in the reactivity were compared at different time intervals $-\leq 7$ days, $8-15$ days, and $\geq 16$ days. To analyze the effect of age on reactivity, a comparison was made between pediatric (6-18 years), adult (19-59 years), and old (60 years and above). Comparison of antibody reactivity was carried out between ICU and non-ICU admissions. Demographic details (age, gender) of the study population, admission date, ward were recorded.

\section{Sample Collection and Laboratory Analysis}

Venous blood $(3 \mathrm{~mL})$ collected by venipuncture under aseptic conditions in clot activator vials wearing personal protective equipment (PPE) was transferred to the clinical biochemistry lab and centrifuged at $3000 \mathrm{rpm}$ for $10 \mathrm{~min}$. Separated serum was used for the analysis of anti-SARSCoV-2 on Roche Cobas 6000 autoanalyzer based on electrochemiluminescence Immunoassay (ECLIA) using Roche Elecsys anti-SARS-CoV-2 kit (Catalogue no09203095190). The data were analyzed in terms of the cut off index (COI) calculated automatically by autoanalyzer based on calibrators- ACOV Cal1 and ACOV Cal2. Subjects were categorized as anti-SARS-CoV-2 reactive and non-reactive based on $\mathrm{COI}$ value. $\mathrm{COI} \geq 1$ was considered reactive, interpreted as positive, and $\mathrm{COI}<1$ was considered non-reactive, interpreted as negative as per manufacturer's instructions.

\section{Statistical Analysis}

Discrete categorical data as age, gender were represented by number, percentage and were compared by using the Chi-square test. Quantitative data were represented as median, interquartile range, minimum and maximum. Kolmogorov-Smirnov test of normality was used to check the normality of quantitative data and groups with sample size $<30$ were considered to be non-parametric. MannWhitney test was used to compare non-parametric data between two groups. The statistical analysis was performed using GraphPad Prism 8.0.2.

\section{Results}

\section{Patient Information}

One hundred and seventy-four serum samples were collected from 103 SARS-CoV-2 patients confirmed by RTPCR admitted in ICU wards and non-ICU wards. These samples were preserved from the samples received in the clinical biochemistry laboratory for routine biochemical analysis. Samples were collected at different time points to study the kinetics of antibody response. There were 79, 64, and 31 samples for the time intervals $-\leq 7$ days, between 8 and 15 days, and $\geq 16$ days respectively. To understand the dynamics of antibody response, serial monitoring of anti-SARS-CoV-2 from 7 patients was done from each of the patients over a period of 1-39 days. Out of 103 patients, forty-six and fifty-seven serum samples were from females and males, respectively. Pediatric, adult, and old age patients contributed to 11,81 , and 11 samples respectively. To understand the antibody kinetics in disease 
severity anti-SARS-CoV-2 response was studied among 21 ICU and 82 non-ICU patients (Fig. 1).

\section{Kinetics of Anti-SARS-CoV-2 in Relation to Time}

In all the 103 patients studied, 62\% (64 patients) were reactive to anti-SARS-CoV-2 and 38\% (39 patients) were non-reactive as shown in Fig. 2a. Roche Elecsys AntiSARS-CoV-2 serological test used in our study detects IgM and IgG antibodies in patient plasma against the nucleocapsid $(\mathrm{N})$ of SARS-CoV-2. To further understand the kinetics of antibody reactivity, anti-SARS-CoV-2 positivity was compared at $\leq 7$ days, $8-15$ days, $\geq 16$ days time intervals. Reactivity to anti-SARS-CoV-2 increased with duration after admission due to COVID-19. Detection of antibodies in 79 samples during the time period of $\leq 7$ days indicated that $33 \%$ (26 samples) were reactive and $67 \%$ (53 samples) were non-reactive (Fig. 2b). As shown in Fig. 2c, between 8 and 15 days, 62\% (40 samples) were reactive and $38 \%$ (24 samples) were non-reactive while the reactivity increased to $81 \%$ (25 samples out of 31 ) in

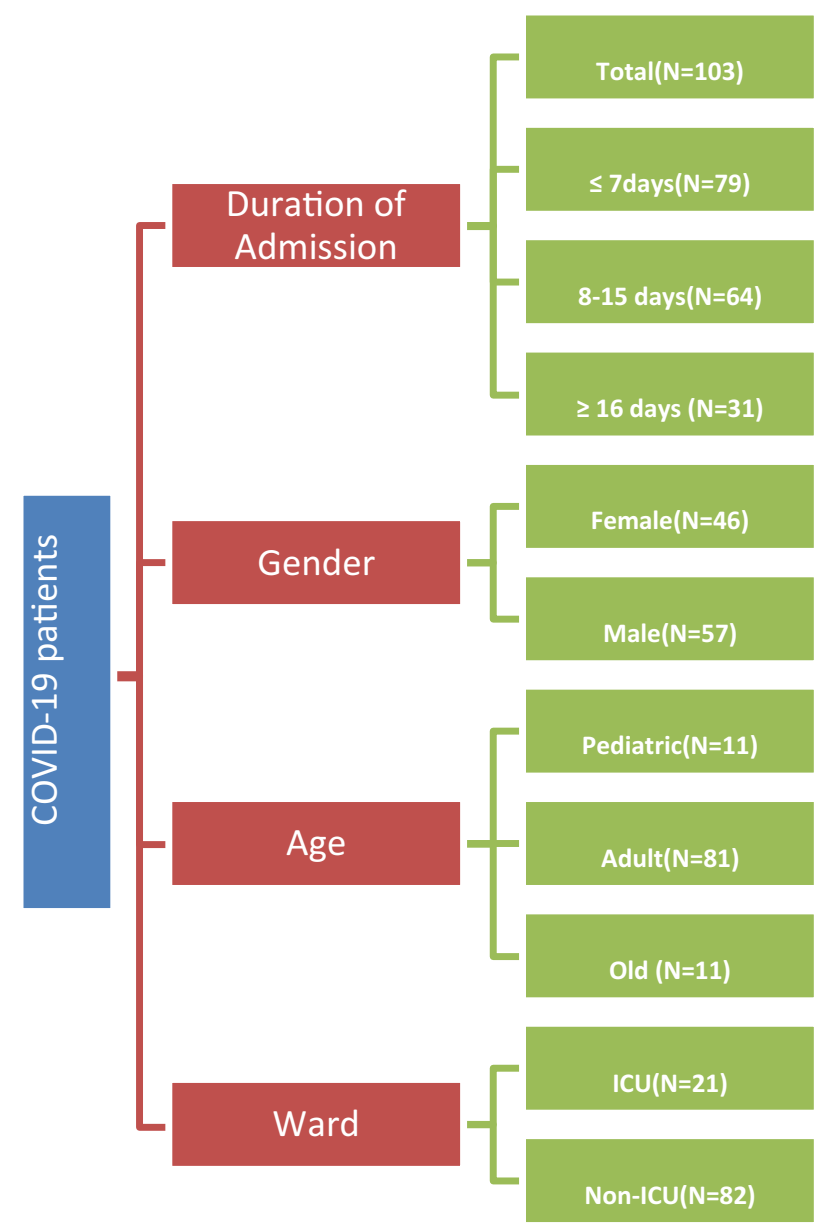

Fig. 1 Categorization of COVID-19 patients recruited in the study the $\geq 16$ days time interval (Fig. 2 d). Thus, there is an increase in the $\operatorname{IgM}$ and $\operatorname{IgG}$ production with time and the maximum response was observed after 16 days.

As shown in Fig. 3, serum levels of anti-SARS-Cov-2 antibodies in terms of COI increased with time. The median value of the antibody reactivity (COI) of positive patients within $\leq 7$ days of admission in hospital was 3.95 (2.03-10.71) which was raised to 7.07 (3.26-12.33) $(p<0.05)$ between 8 and 15 days. Antibody reactivity (COI) was 13.85 (8.28-23.02) for the samples from positive patients after $\geq 16$ days of admission to the hospital which was significantly increased as compared to $\leq 7$ days $(p<0.0001)$ and $8-15$ days $(p<0.01)$ groups.

\section{Alterations in Serological Response of COVID-19 Patients in Relation to Gender and Age}

As observed in Fig. 4a, more men $(n=57)$ were diagnosed with COVID-19 than women $(n=46)$ in the recruited study group. Serological response varied among men and women with a percentage positivity of 65 , and $59 \%$ in males and females, respectively indicating more men had developed antibody response against SARS-Cov-2.

Among pediatric $(\mathrm{n}=11)$, adult $(\mathrm{n}=81)$, and old $(\mathrm{n}=11)$ age populations studied, not much difference was observed in terms of the antibody response as reactivity observed was in 62,64 , and $62 \%$ respectively (Fig. 4b).

\section{Anti-SARS-CoV-2 Reactivity Patterns Vary in the COVID-19 Patients}

Reactivity of seven individual patients was studied at different time points after the positive RT-PCR test for COVID-19 to understand the dynamics of antibody response. As shown in Fig. 5, only three out of seven subjects produced antibodies by the 7 th day $(\mathrm{COI}>1)$, and the number increased to five by the 14th day. Two of the seven COVID 19 patients could not elicit antibody response till around 20 days of diagnosis (subject numbers 6 and 7). The pattern of antibody reactivity in terms of COI varied among individuals. The antibody production increased as days progressed even up to 39 days for subject 4 while it showed plateau from as early as day 7 till day 25 in subject 2. On day 20 post-admission, anti-SARS-CoV-2 levels (COI) in subject 3 was 45.3 while it was 19.45 in subject 5 again suggesting varied antibody responses among individuals.

\section{COVID-19 Severity is Associated with Different Anti-SARS-CoV-2 Response}

Antibody response was analyzed among 21 patients admitted in ICU and 82 patients admitted in non-ICU 
(A)
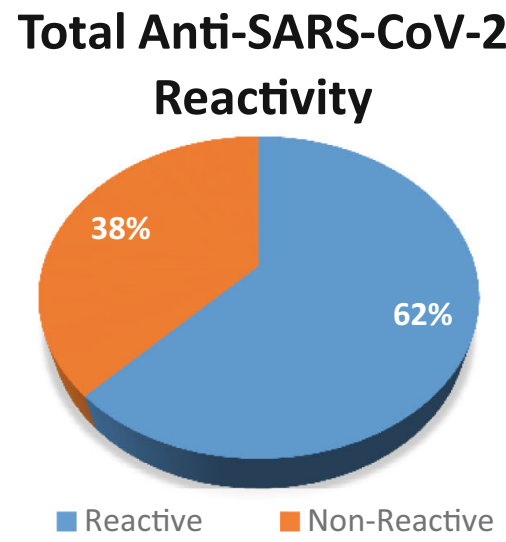

(C)
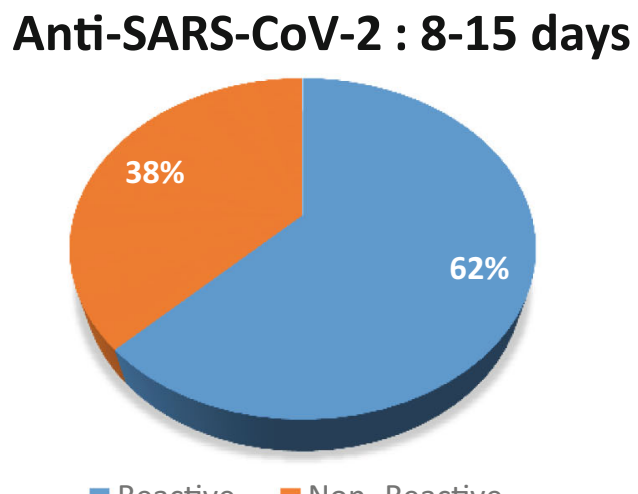

Reactive Non- Reactive

\section{(B) Anti-SARS-CoV-2 : $\leq 7$ days}

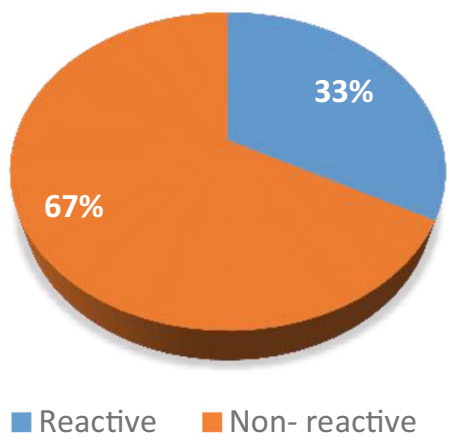

(D) Anti-SARS-CoV-2: $\geq 16$ days

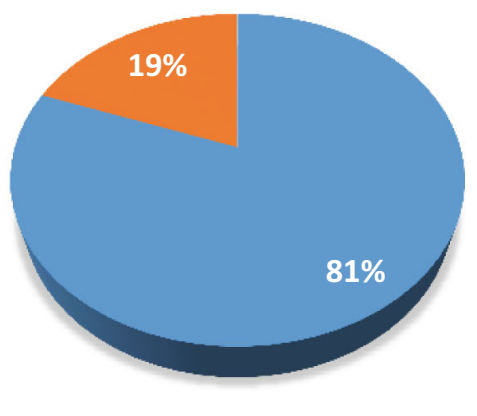

Reactive Non-Reactive

Fig. 2 Relative proportion of anti-SARS-CoV-2 reactivity in COVID-19 patients. The proportion of reactive and non-reactive at a Total (entire duration), $\mathbf{b} \leq 7$ days, $\mathbf{c} 8-15$ days, and $\mathbf{d} \geq 16$ days from the day of admission

wards. As shown in Fig. 6a, percentage antibody reactivity was higher in patients admitted in ICU with $67 \%(\mathrm{n}=14$ out of 21) being positive as compared to $60 \%$ ( $n=49$ out of 82) positivity in non-ICU wards patients. In the antiSARS-CoV-2 positive ICU and non-ICU patients, median COI (IQR) was 3.90 (2.03-10.16) and 8.78 (3.54-13.85) respectively (Fig. 6b) which was not significantly different.

\section{Discussion}

The kinetics of combined $\operatorname{IgM}$ and $\operatorname{IgG}$ response to nucleocapsid of SARS-CoV-2 in 103 serum samples of COVID-19 patients were studied to understand the immunological response among females and males, in relation to age, duration of infection, and disease severity. As compared to females, more males were affected with COVID-19 as observed in a previous study also [5]. In our study, the percentage positivity for antibody (IgM and $\operatorname{IgG}$ ) in males $(65 \%)$ was higher as compared to females $(59 \%)$ which are in contrast to a previous study wherein antibody response (IgG) was higher in females with severe COVID19 [10]. The difference in the observation might be due to the detection of the only IgG in the study by Fanfan Zeng et al. [10] as compared to both IgG and IgM in the present study. Studies showed that SARS-CoV-2 elicits a serological response in children, adult, and old subjects [5, 10, 12]. In our study, an almost similar percentage of COVID-19 adult (62\%), pediatric (64\%), and old patients (64\%) were seropositive. Although, the immune responses, in general, are known to differ in different age groups [11], however, the limitation of our finding is that we had less number of patients in pediatric and old age groups $(n=11$ each).

The production of IgM peaks in the second week and that of $\operatorname{IgG}$ in the third week post-infection due to class switch [13]. Anti-SARS-CoV-2 (IgM and IgG) reactivity increased with time, from $33 \%$ positivity at $\leq 7$ days to $62 \%$ positivity between 8 and 15 days, the highest being $81 \%$ at $\geq 16$ days after hospital admission. This gradual increase in positivity might be due to increased IgM production later associated with isotype class switch to $\mathrm{IgG}$, 


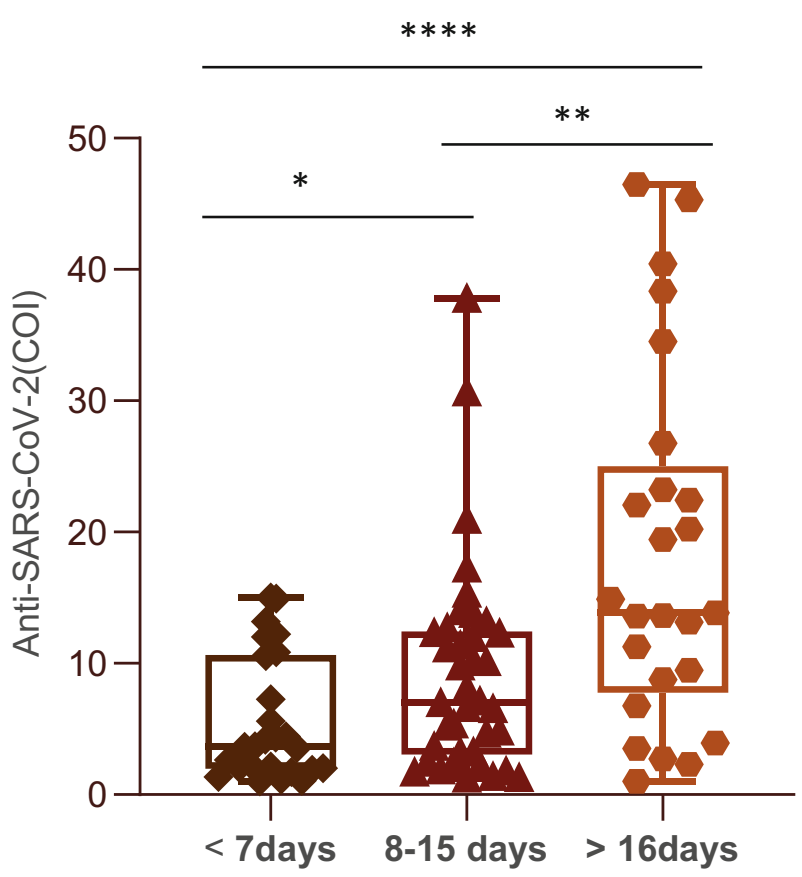

Fig. 3 Box plot representation of anti-SARS-CoV-2 reactivity (COI) at $\leq 7$ days, $8-15$ days, and $\geq 16$ days after diagnosis of COVID-19. The interquartile range is represented by the length of the box, individual COI values are represented by solid shapes inside the box, the line in the box represents the median, the vertical lines extend from the box to maximum and minimum values. (**** $p<0.0001$ for comparison between $\leq 7$ days and $\geq 16$ days, ${ }^{*} p<0.05$ for comparison between $\leq 7$ days and 8-15 days and $* * p<0.01$ for comparison between 8 and 15 days and $\geq 16$ days)

thus collectively leading to an increased immunological response. SARS-CoV-2 failed to develop antibody response in all the patients as total anti-SARS-COV-2 positivity was $62 \%$ considering all the patients. The failure to produce antibodies could be due to underlying immunocompromised states. Anemia and disseminated

(A)

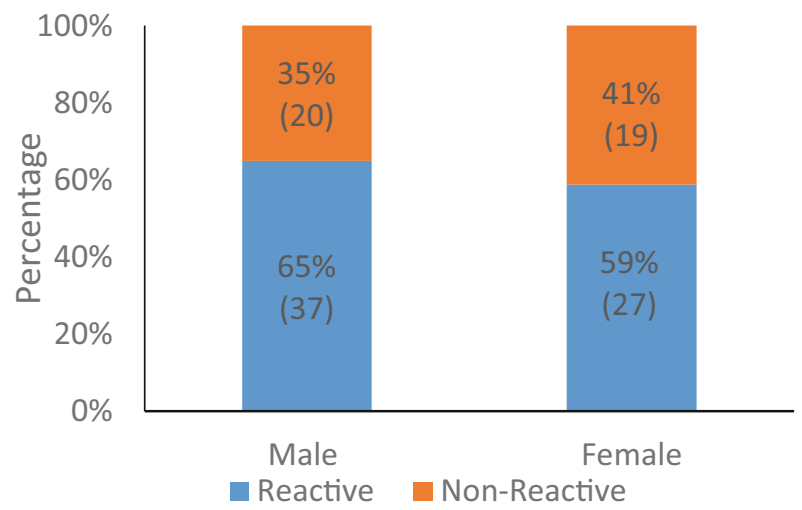

Fig. 4 Anti-SARS-CoV-2 reactivity in COVID-19 patients. a Percentage comparison of reactive and non-reactive patients in male and female groups (numbers in the bracket represent patients count) and tuberculosis were observed in two of the patients with antiSARS-CoV-2 negativity in our study. However, we were unable to procure the previous medical history of all our study population.

Maximum antibody production was observed at $\geq 16$ days of admission into the hospital with anti-SARS-CoV-2 (COI) of 13.85, which was significantly higher than antibody production at $\leq 7$ days and between 8 and 15 days. The increased antibody production at this time frame could be because of $\mathrm{B}$ cell clonal expansion and extensive class switching from IgM to IgG [14]. Trends in the rise of antibody production altered from person to person. Maximum reactivity for anti-SARS-CoV-2 was observed at a different time in different individuals studied. A patient with less severe COVID-19 not requiring ventilation was monitored till the 39th day after admission. There was a gradual increase in reactivity, but the patient had an antiSARS-CoV-2 value lesser than the other 3 ICU reactive patients at a given time. Lesser reactivity may be because the mild infection is associated with less viral load leading to lesser antibody production [15]. A constant level of antibody production was seen in a patient who was a known hypertensive. Two of the COVID-19 patients were anti-SARS-COV-2 non-reactive, one of them was admitted in the ICU ward requiring ventilation, and the other in nonICU without ventilation. Thus, antibody response does not depend only on the viral load, it also depends on the host immune response. In a previous study, COVID-19 patients' failure to initiate antibody response was associated with leukemia and lymphoma [6]. Any history of previous illness in these patients is lacking in our study. Percentage of anti-SARS-CoV-2 positivity (COI $>1$ ) was higher in ICU (67\%) as compared to non-ICU (60\%), which could be due to high viral load in ICU patients during the initial period [16]. The antibody production, however, was more in non-

(B)

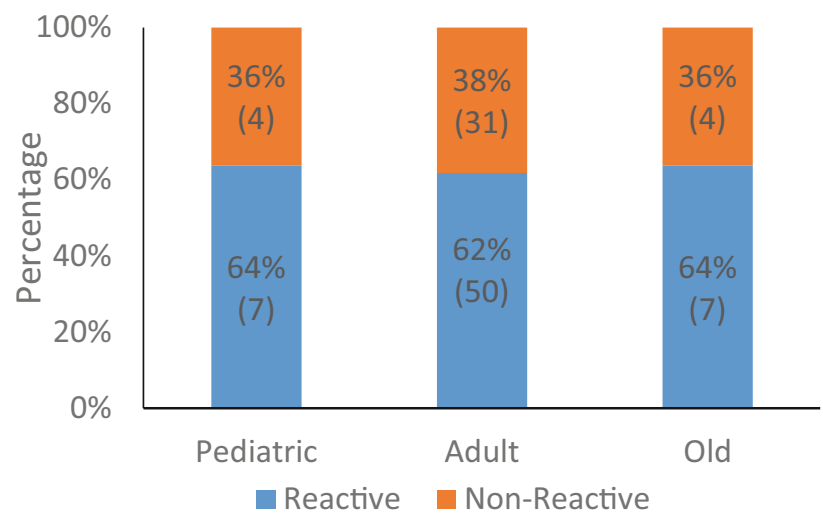

b Percentage comparison of reactive and non-reactive in pediatric, adult, and old age groups (numbers in the bracket represent patients count) 


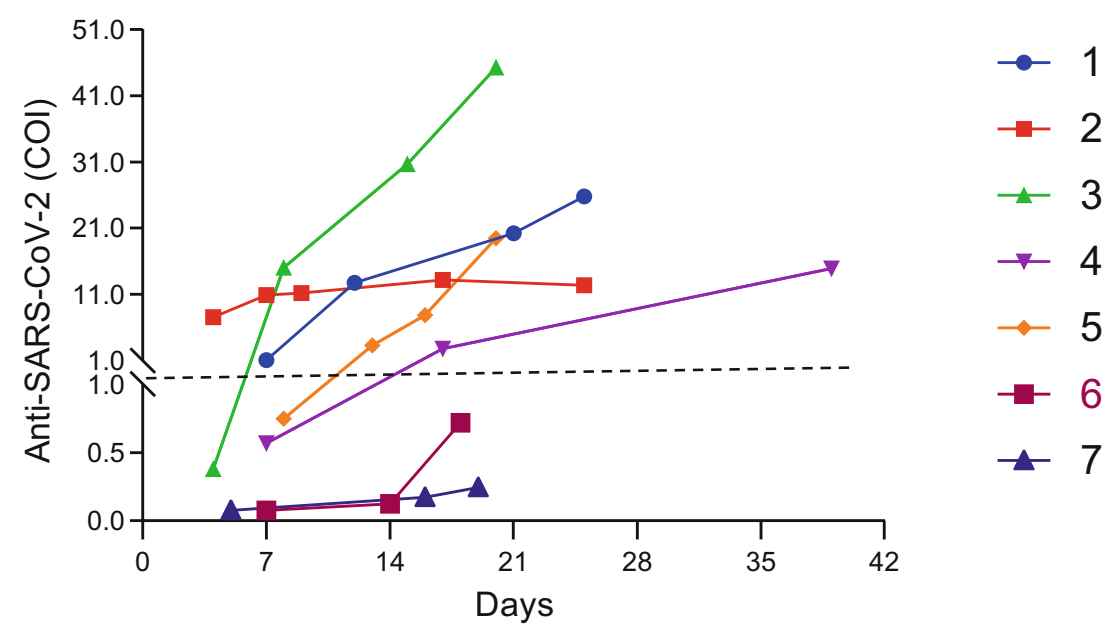

Fig. 5 The line graph representation of anti-SARS-CoV-2 reactivity (COI) in seven COVID-19 patients in relation to time from the day of admission. Each solid shape represents one particular patient with COI at different time points, connected by the solid line. The dotted

ICU patients with an anti-SARS-COV-2 (COI) value of 8.78 as compared to ICU patients with a value of 3.90 which may be due to adequate antibody production in nonICU patients in an early phase of infection enabling them to control the disease, thereby decreasing the severity. These findings concur with previous studies on the role of disease severity in antibody response in COVID-19 [15, 17].

In conclusion, the profile and kinetics of antibody response during COVID-19 disease are dependent on

(A)

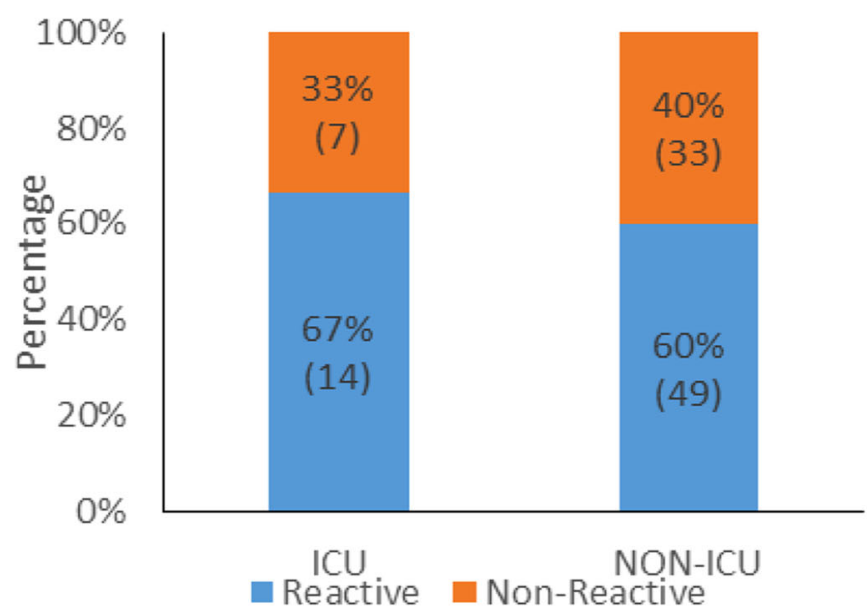

Fig. 6 Anti-SARS-CoV-2 reactivity of COVID-19 patients in ICU and Non-ICU wards a Percentage comparison of reactive and nonreactive patients admitted in ICU and Non-ICU wards (Number in the bracket represent patients count) and $\mathbf{b}$ Box plot representation of anti-SARS-CoV-2 reactivity (COI) in ICU and Non-ICU patients. The line represents $\mathrm{COI}=1$, the cut off for reactivity. 5 patients are reactive and all of them are reactive between 7 and 14 days. 2 patients are non-reactive even nearing 21 days from the day of admission

gender, age, disease severity, and host immune response. Hence, during the use of plasma from the convalescent donor for passive immunization, estimation of antibodies becomes necessary as it varies among patients. Initiation of antibody production varies from person to person, therefore usage of serological test for diagnosis of COVID-19 has a limited value. Thus, understanding the kinetics of antibody production in SARS-COV-2 infection can help in better treatment, monitoring of COVID-19 patients, vaccine

(B)

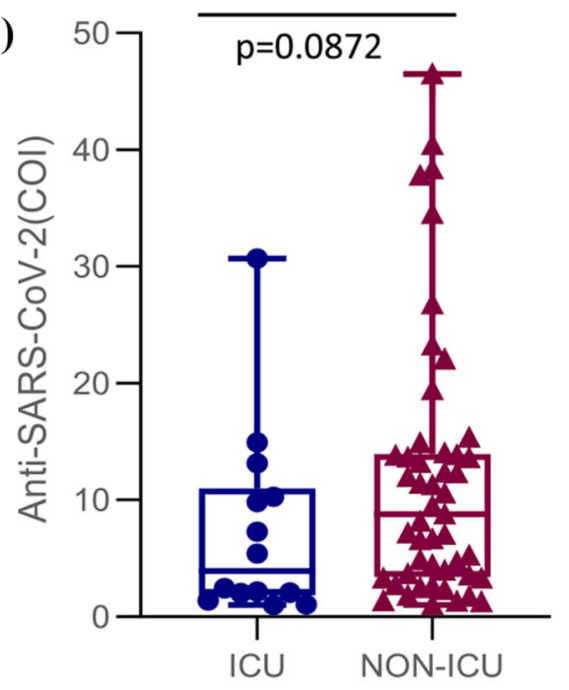

interquartile range is represented by the length of the box, individual values are represented by solid shapes inside the box, line in the box represents the median, the vertical lines extend from the box to maximum and minimum values 
development, and epidemiological investigations for the control of COVID-19. Small sample size with unequal sample numbers between the study groups and lack of information of co-morbid conditions are some of the limitations of this study.

Authors' Contributions MKC- Writing original draft, investigation, formal data analysis and interpretation. DZ- Methodology, conceptualization (supplementary) of the study. SR- Methodology, conceptualization (supplementary) of the study. SLS- Recuirtment, management and sampling of patients. VS- Recuirtment, management and sampling of patients. PM- Recuirtment, management and sampling of patients. RSJ- Investigation of samples. JK- Project administration, lead conceptualization and designing of the study, formal analysis, interpretation, review and editing of manuscript. IV- Project administration, lead conceptualization and designing of the study, formal analysis, interpretation, review and editing of manuscript. SSProject administration, conceptualization (supplementary) of the study.

Funding No funding was obtained from any organization.

\section{Declarations}

Conflicts of Interest The Authors declares that they have no conflict of interest.

Ethical Approval Study was approved by Institute Ethics Committee (Ref No-NK/6384/study/231).

\section{References}

1. Harapan H, Itoh N, Yufika A, Winardi W, Keam S, Te H, et al. Coronavirus disease 2019 (COVID-19): a literature review. J Infect Public health. 2020;13:667-73.

2. Li F. Structure, function, and evolution of coronavirus spike proteins. Ann Review Virol. 2016;29(3):237-61.

3. Wang Y, Zhang L, Sang L, Ye F, Ruan S, Zhong B, et al. Kinetics of viral load and antibody response in relation to COVID-19 severity. J Clin Investig. 2020;130(10):5235-44.

4. Wang L, Wang Y, Ye D, Liu Q. Review of the 2019 novel coronavirus (SARS-CoV-2) based on current evidence. Int $\mathbf{J}$ Antimicrob Agents. 2020;55(6):105948.
5. Liu D, Cui P, Zeng S, Wang S, Feng X, Xu S, et al. Risk factors for developing into critical COVID-19 patients in Wuhan, China: a multicenter, retrospective, cohort study. EClinicalMedicine. 2020;25:100471.

6. Winter AK, Hegde ST. The important role of serology for COVID-19 control. Lancet Infect Dis. 2020;20(7):758-9.

7. Rode OĐ, Kurolt IC, Puljiz I, Balent NC, Laškaj R, Tiljak MK, et al. Antibody response and the clinical presentation of patients with COVID-19 in Croatia: the importance of a two-step testing approach. Eur J Clin Microbiol Infect Dis. 2021;40(2):261-8.

8. To KK, Tsang OT, Leung WS, Tam AR, Wu TC, Lung DC, et al. Temporal profiles of viral load in posterior oropharyngeal saliva samples and serum antibody responses during infection by SARS-CoV-2: an observational cohort study. Lancet Infect Dis. 2020;20(5):565-74.

9. Zhao J, Yuan Q, Wang H, Liu W, Liao X, Su Y, et al. Antibody responses to SARS-CoV-2 in patients with novel coronavirus disease 2019. Clin Infect Dis. 2020;71(16):2027-34.

10. Zeng F, Dai C, Cai P, Wang J, Xu L, Li J, et al. A comparison study of SARS-CoV-2 IgG antibody between male and female COVID-19 patients: a possible reason underlying different outcome between sex. J Med Virol. 2020;92(10):2050-4.

11. Simon AK, Hollander GA, McMichael A. Evolution of the immune system in humans from infancy to old age. Proc R Soc B Biol Sci. 2015;282(1821):20143085.

12. Jia R, Wang $X$, Liu $P$, Liang $X$, Ge $Y$, Tian $H$, et al. Mild cytokine elevation, moderate $\mathrm{CD} 4+\mathrm{T}$ cell response and abundant antibody production in children with COVID-19. Virol Sinica. 2020;22:1-10.

13. Xiang F, Wang X, He X, Peng Z, Yang B, Zhang J, et al. Antibody detection and dynamic characteristics in patients with coronavirus disease 2019. Clin Infect Dis. 2020;71(8):1930-4.

14. Nielsen SC, Yang F, Jackson KJ, Hoh RA, Röltgen K, Jean GH, et al. Human B cell clonal expansion and convergent antibody responses to SARS-CoV-2. Cell Host Microbe. 2020;28(4): 516-25.

15. Liu X, Wang J, Xu X, Liao G, Chen Y, Hu CH. Patterns of IgG and IgM antibody response in COVID-19 patients. Emerg Microbes Infect. 2020;9(1):1269-74.

16. Sun B, Feng Y, Mo X, Zheng P, Wang Q, Li P, et al. Kinetics of SARS-CoV-2 specific IgM and IgG responses in COVID-19 patients. Emerg Microbes Infect. 2020;9(1):940-8.

17. Liu L, To KK, Chan KH, Wong YC, Zhou R, Kwan KY, et al. High neutralizing antibody titer in intensive care unit patients with COVID-19. Emerg Microbes Infect. 2020;9(1):1664-70.

Publisher's Note Springer Nature remains neutral with regard to jurisdictional claims in published maps and institutional affiliations. 